\title{
Coughs cause systemic blood flow
}

\author{
JEFFREY M CARY, RICHARD KRUGMEIER, BILL NEWMAN, BRIAN ROSS, JOHN \\ BUTLER
}

From the Departments of Medicine and Surgery, University of Washington, Seattle, USA

\begin{abstract}
Although it is known that rhythmic coughing can preserve consciousness during ventricular fibrillation, the arterial pressure transients which result have not yet been shown to reflect forward blood flow in man. The effectiveness of cough in causing foward flow in eight normal volunteers with bradycardia was studied. They coughed between cardiac cycles, using an audio and visual display of the electrocardiogram. The force of the cough was varied and measured with an oesophageal balloon. Blood flow was recorded with a Döppler velocity probe over the radial artery and a finger or ear photoplethysmograph. Motion artefact on the Döppler record due to coughing was excluded by transiently obstructing the brachial artery. We compared the areas under 5-10 consecutive Döppler and photoplethysmograph pulse flow tracings due to the cough and heart beat with those due to the immediately preceding heart beat alone. They were significantly increased. This augmentation was greater in those flow pulses accompanied by a more vigorous cough. It is concluded that coughing is associated with an effort dependent foward flow pulse in the arterial circulation.
\end{abstract}

Kowenhoven et al' were the first to report that blood could be made to flow out of the closed chest by externally applied pressure to the chest wall. They proposed that this blood flow was brought about by squeezing the heart between the sternum and the spine. This "external cardiac massage" was accepted as the basis of cardiopulmonary resuscitation by the American Heart Association in $1974^{2}$ and the mortality rate of sudden cardiac arrest appears to have been dramatically reduced at the same time that rapidly administered cardiopulmonary resuscitation by trained bystanders became available. There are even indications that the early efforts at external chest compression by the lay population may be more successful than the cardiopulmonary resuscitation administered later by trained personnel. ${ }^{3}$ The implication of this observation is that rapid reestablishment of vascular perfusion is essential for successful resuscitation. It also suggests that it may not be the specific method of chest compression but rather the development of positive intrathoracic pressure that produces the blood flow. Criley et al have shown that patients developing ventricular

Address for reprint requests: Professor John Butler, Division of Respiratory Diseases RM-12, University of Washington, Seattle, Washington 98195, USA.

Accepted 21 November 1983 fibrillation during cardiac catheterisation may carry out their own positive intrathoracic pressure resuscitation and maintain consciousness by coughing in a forceful, rhythmic fashion. ${ }^{4}$ In his study, patients developed peak arterial systolic pressures of about $140 \mathrm{~mm} \mathrm{Hg}$ during coughing. This and the prolongation of consciousness suggested that flow to the brain was preserved. It has been known, however, since the studies of Weale and Rothwell-Jackson ${ }^{5}$ that there is an equal intrathoracic increase in venous and arterial pressures during chest compression. Thus the presence of an arterial pulse during coughing does not necessarily reflect a forward blood flow, although the preservation of consciousness suggests it. We designed a study to evaluate the effectiveness of coughing in man using non-invasive methods for assessing blood flow at different anatomical sites and correlating the intrathoracic pressure with the measured blood flow.

\section{Methods}

Eight men aged 21-45 years were selected because they had considerable bradycardia secondary to athletic training. The subject observed the electrocardiogram displayed on an oscilloscope and listened to a sound triggered by the QRS complex. An oesophageal balloon was passed to the distal oesophagus. ${ }^{6}$ Blood flow was measured by two 


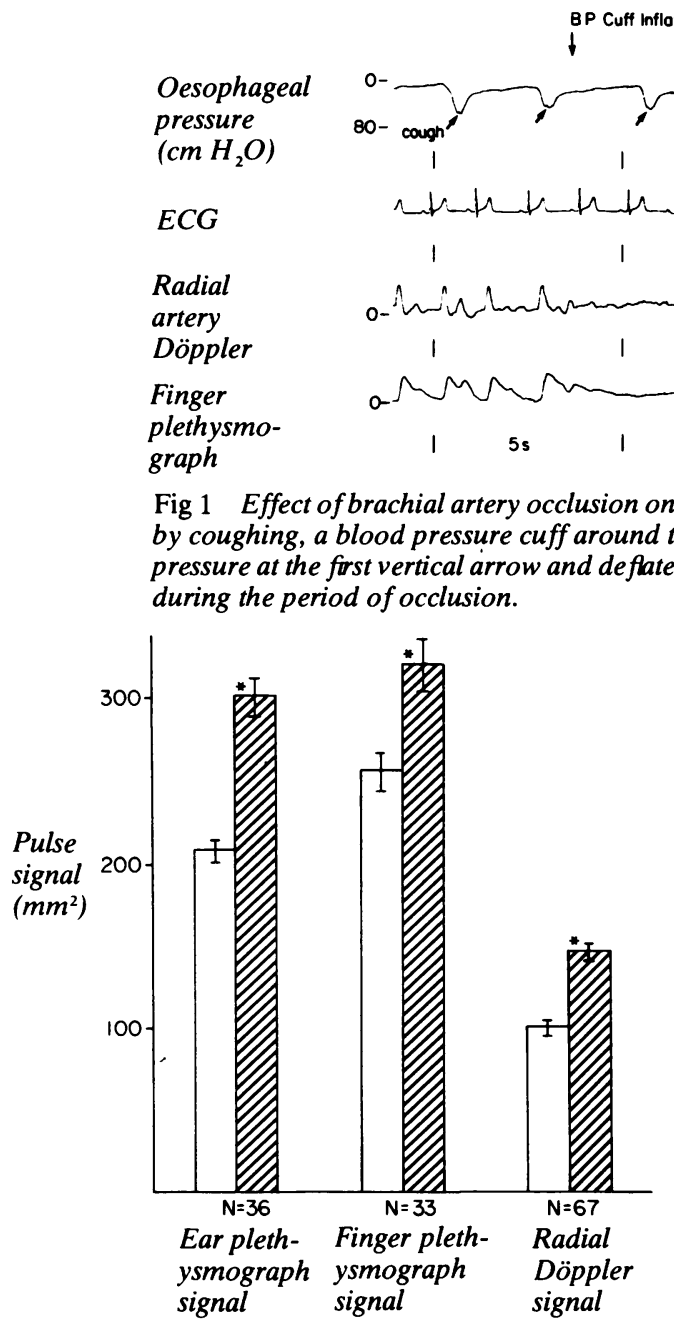

Fig 2 Flow due to cardiac pulse alone contrasted with that due to the succeeding pulse augmented by cough (means and standard errors). A radial artery Döppler display was always used; in about half the studies the

photoplethysmograph was used on the ear and in the rest on a finger. $\square$ cardiac pulse; cardiac pulse plus cough. ${ }^{*} p<0.001$.

devices: a Döppler velocity probe over the radial artery and a finger or ear photoplethysmograph. ${ }^{7}$ The Döppler velocity meter transmits a continuous beam of ultrasound through the skin, subcutaneous tissue, and vessel wall into the passing blood stream. The ultrasonic signal reflected from the moving particles in the blood is shifted in frequency in proportion to the flow velocity. The instrument produces a signal proportional to instantaneous blood flow. Volume flows at the same position may be compared if it is assumed that velocity profiles and vessel calibre do not change. In the photoplethysmograph light is transmitted through a capillary bed and detected by a photosensor. Changes in the volume of the capillary bed between the light source and the photosensor modify absorption of the light. The rate of change of volume of the capillary bed between the light source and the photosensor reflects arterial inflow. These methods allow comparison of pulse volumes with the normal (control) pulse but were not calibrated in flow units.

The subjects lay in the supine or, to minimise possible oesophageal pressure artefacts, the lateral position. They were taught to cough by executing a brief Valsalva manoeuvre against a closed glottis after a tidal inspiration and practised increasing their effort using an oscilloscopic display of the oesophageal pressure. Then they coughed immediately after QRS complexes of random cardiac cycles, with normal effort initially and then with increasing or decreasing vigour in a randiom fashion.

To assess motion artefact in the Döppler and plethysmographic recordings during the coughing manoeuvres the brachial artery was occluded by inflating a blood pressure cuff around the upper arm to above systolic pressure. The subjects continued to cough and the radial artery Döppler and finger plethysmographic signals were followed.

The effect of five to 10 consecutive coughs on the Döppler and plethysmographic signals was analysed by comparing the area under the pulse signal generated by the sum of the cough and the cardiac action with the area under the immediately preceding pulse signal generated by the cardiac action alone. Timing and technical artefact prevented some measurements, but about the same number of coughs were analysed for each subject.

Coughs during systole were excluded. The flow signal areas obtained by planimetry reflected pulse volumes and were compared with a paired $t$ test. 


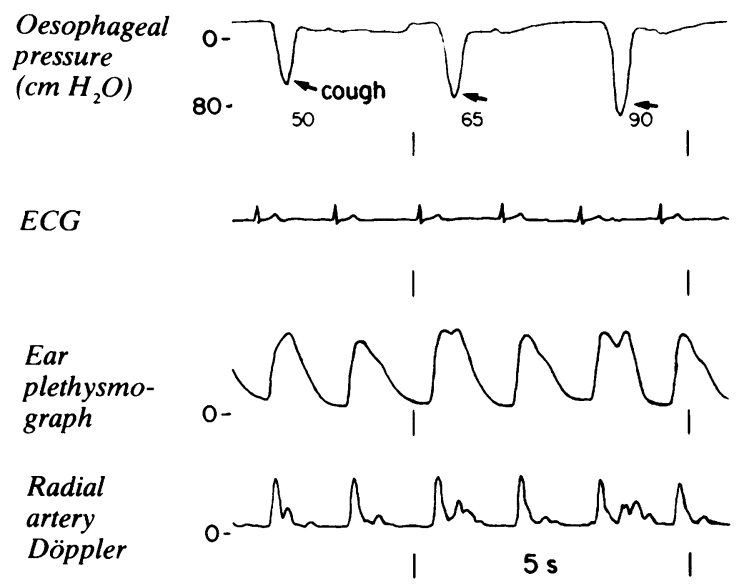

Fig 3 Effect of cough effort on pulse signal. Coughs of increased vigour with oesophageal pressures of 50,65, and $90 \mathrm{~cm} \mathrm{H}_{2} \mathrm{O}$ caused progressively greater flows.

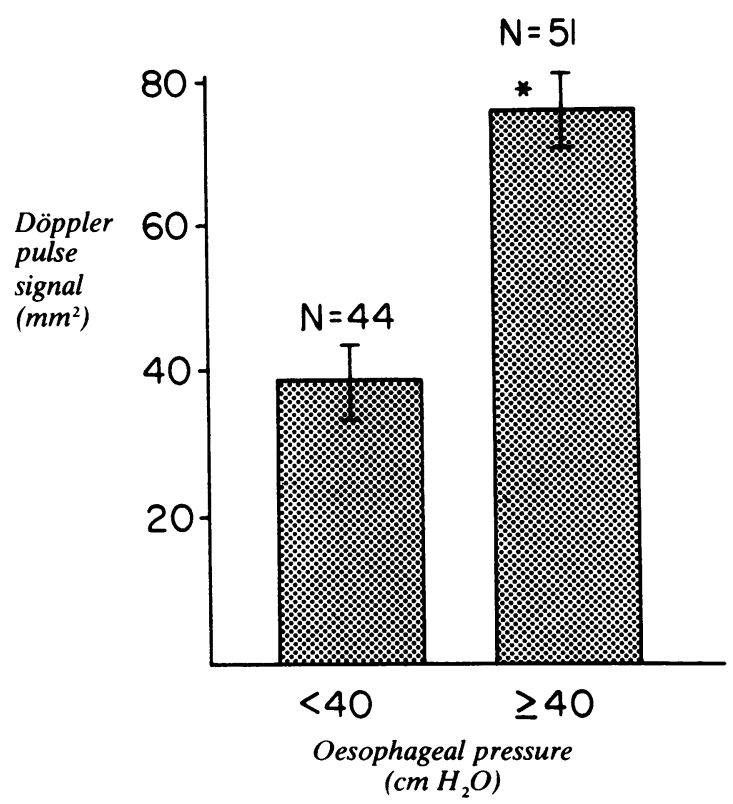

Fig 4 Blood flow (means and standard errors) assessed by Döppler pulse signal for coughs producing pressures greater than and less than $40 \mathrm{~cm} \mathrm{H}_{2} \mathrm{O} .{ }^{*} \mathrm{p}<0.001$.

\section{Results}

There was little motion artefact in the radial artery Döppler or finger plethysmograph tracing when blood flow to the arm was prevented by inflation of the blood pressure cuff (fig 1). When the cuff was released the expected flow signals from the cardiac cycles and the cardiac cycles plus cough returned,

The ear photoplethysmograph, the finger photoplethysmograph, and the radial artery Döppler data showed that the pulse volumes generated by cough plus cardiac action were significantly greater than those due to cardiac action alone (fig 2). Any positiion artefact was minimised by the comparison with the pulse due to cardiac action alone which immediately preceded that due to cardiac action plus cough. We did not find an effect of the diastolic timing of the cough on its flow augmentation or on the succeeding pulse.

The augmentation of blood flow was related to the effort and positive pressure developed during the cough (fig 3). Since it proved impossible to standardise the oesophageal pressures reached during coughing, the pulse volumes generated by coughs with oesophageal pressures greater than $40 \mathrm{~cm}$ of water were compared with those when pressures were less than $40 \mathrm{~cm}$ of water. There were significantly greater pulse volumes produced by the more vigorous coughing (fig 4).

\section{Discussion}

The time and vigour of coughing were analysed on the basis of oesophageal pressure. When this is measured in the supine position, changes during breathing may not reflect intrathoracic pressure changes. These errors seem to be related to changes of lung volume, which may displace the organs and alter the weight of the mediastinum on the oesophagus. In our studies lung volume during the cough (functional residual capacity plus tidal volume) was not altered, so such artefacts are unlikely, particularly since the results of studies of the relationship between pressure and pulse signal in subjects in the lateral decubitus position were not different from those in the supine position. Motion due to cough did not affect the Döppler arterial velocity trace. Although sustained changes in intrathoracic pressure are reflected in the veins, venous backflow transients which might have affected the photoplethysmograph are probably prevented by venous valves. ${ }^{8}$

Our normal subjects with sinus bradycardia coughed during diastole when the aortic valve was closed. This is the time when cardiac assist devices such as the aortic balloon pump are used. It must be emphasised that their cardiovascular state was very different from that of patients with ventricular fibrillation. Yet our study indicates that the systemic 
pressure pulse, known to be produced by coughing in these patients and associated with the preservation of consciousness, is associated with a forward flow pulse in the periphery. As would be expected if intrathoracic pressure was the cause of the forward pulse, the flow pulses were greater when the oesophageal pressures were higher. This confirms in man the direct relationship between intrathoracic pressure and flow reported by Rosborough et al in anaesthetised dogs with simulated coughs produced with the aid of an "external glottis."

The mechanism of this foward flow is thought to be the difference between the arterial and venous pressures outside the chest during the positive intrathoracic pressure phase. This pressure, unable to squeeze air out of the chest because of the closed glottis, squeezes out blood from the lungs, left heart, and aorta. Venous backflow regurgitation appears to be limited both by venous valves and by collapse of the veins at the thoracic outlet caused by the positive intrathoracic pressure transients. ${ }^{8}$ Consequently, a driving pressure exists between the arteries and veins outside the chest during the cough and forward flow occurs.

\section{References}

' Kowenhoven WB, Jude JR, Knickerbocker GG. Closedchest cardiac massage. JAMA 1960;173:1064-7.

${ }^{2}$ American Heart Association. Standards for cardiopulmonary resuscitation (CPR) and emergency cardiac care (ECC). JAMA 1974;227:833-68.

${ }^{3}$ Cobb LA, Werner JA, Trobaugh GB. Sudden cardiac death. ii-Outcome of resuscitation: management and future directions. Modern Concepts of Cardiovascular Disease 1980;49:37-40.

${ }^{4}$ Criley JM, Blaufuss AH, Kissel GL. Cough induced cardiac compression: self administered form of cardiopulmonary resuscitation. JAMA 1976;236:124650.

${ }^{5}$ Weale FE, Rothwell-Jackson RL. The efficiency of cardiac massage. Lancet 1962;1:990-2.

${ }^{6}$ Milic-Emili J. Mead J, Turner JM, Glauser EM. Improved technique for estimating pleural pressure from esophageal balloons. J Appl Physiol 1966;19:207-11.

7 Verstraete M, ed. Instrumentation and techniques in clinical medicine. Vol 2. Boston: Martinus Nijohoff, 1980:43-5, 112-8.

${ }^{8}$ Rudikoff MT, Maughan WL, Effoon M, Freund P, Weisfeldt ML. Mechanisms of blood flow during cardiopulmonary resuscitation. Circulation 1980; 61:345-52.

${ }^{9}$ Rosborough JP, Hausnecht M, Niemann JT, Criley JM. Cough supported circulation. Crit Care Med 1981;9:371. 